\title{
Identification of initial drift in semiconductor gas sensors caused by temperature variation
}

\begin{abstract}
Metal-oxide (MOX) gas sensors are well-known with their high sensitivity in detecting gases. Despite of this quality, the responses of gas sensor are inclined to substantial drift effects caused by the environmental variable of the surrounding atmosphere such as temperature. The variation of temperature introduced shifts in the dynamic features of sensor resistance, and nonlinearly modified the original unique patterns of acquired response. In this paper, the initial drift in MOX gas sensors were identified from the responses of gas sensors that shifted over temperature variation. This initial drift identification is important for further analyses on drift compensation.
\end{abstract}

Keyword: Gas sensor; Response shift; Sensor drift; Temperature 